\title{
Electrolyte Solution Structure and Its Effect on the Properties of Electric Double Layers with Surface Charge Regulation
}

\author{
R. Vangara, D. C. R. Brown, F. van Swol*, D. N. Petsev*
}

Department of Chemical and Biological Engineering, and Center for Micro-Engineered Materials, University of New Mexico, Albuquerque, NM 87131

\begin{abstract}
The physical origin of charged interfaces involving electrolyte solutions is in the thermodynamic equilibrium between the surface reactive groups and certain dissolved ionic species in the bulk. This equilibrium is very strongly dependent on the precise local density of these species, also known as potential determining ions in the solution. The latter, however, is determined by the overall solution structure, which is dominated by the large number of solvent molecules relative to all solutes. Hence, the solvent contribution to the molecular structure is a crucial factor that determines the properties of electric double layers. Models that explicitly account for the solvent structure are often referred to as "civilized" as opposed to the "primitive" ones that consider the solvent as a structureless continuum. In the present paper, we demonstrate that for a physically correct description of charged interfaces that involve electrolyte solutions (electric double layers), the full solution structure needs to be taken into account in conjunction with the precise surface chemistry governed by the thermodynamic equilibrium. The analysis shows how the surface charge depends on various experimentally relevant parameters, many of which are outside the realm of simple electrostatics. We present results on the effects of solvent molecular dimensions, ionic solvation, surface chemistry, solvophilicity and solvophobicity.
\end{abstract}

\section{Introduction}

Interfaces involving electrolyte solutions are common in nature as well as in various fields of fundamental and applied research. The contact surface between the electrolyte solution and the second phase is usually charged, leading to a spatial redistribution of ions historically known as an electric double layer (EDL). The surface charge is due to an externally applied potential in the case of metal electrode or to specific surface chemistry in the case of dielectric materials. The EDL is a key concept pertinent to important areas such as colloid and interface science, electrochemistry, corrosion, material science, etc. Therefore it is not surprising that EDLs have been extensively studied for more than a century and the efforts to obtain deeper insights continue at the present time. The first attempt to provide a physical interpretation of the EDL was offered by Helmholtz [1], who considered the charge redistribution at the interface as a simple capacitor. Later it was realized that this is not an accurate representation because of the diffuse distribution of ions in the vicinity due to the thermal motion. A better model, proposed independently by Gouy [2] and Chapman [3], described the ionic distribution using a continuum approximation known as the Poisson-Boltzmann (PB) equation [4]

$$
\nabla^{2} \Psi=-\frac{1}{\varepsilon \varepsilon_{0}} \sum_{i} \rho_{i}^{0} q_{i} \exp \left(\frac{-q_{i} \Psi}{k_{B} T}\right)
$$

Email addresses: vanswol@unm.edu (F. van Swol), dimiter@unm.edu (D. N. Petsev) where $\Psi$ is the electrostatic potential, $q_{i}$ is the charge of ion species " $i$ " (in units of the elementary charge $e$ ), $k_{B} T$ is the thermal energy, $\rho_{i}^{0}$ is the bulk number density of charged species $i, \varepsilon$ and $\varepsilon_{0}$ are the medium dielectric permittivity, and the dielectric constant of vacuum. The Gouy-Chapman approach does not explicitly account for the ionic size and associated correlations in the EDL, which is problematic near the charged interface. This issue was first addressed by Stern [5] and Graham [6] who suggested the presence of a finite sized layer of ions and solvent molecules adjacent to the surface. This layer acts as a capacitor in addition to the diffuse potential determined by the PB equation (1). The idea was further developed by other authors [7-11] who offered various modifications of the PB equation in an attempt to improve the description of the EDL.

More rigorous analyses of EDLs are based on modern statistical mechanics [12-14]. These approaches are usually based on the Ornstein-Zernike (OZ) integral equation or classical density functional theory (DFT) [15-20]. Often their implementation is limited to explicitly accounting only for the charged ionic species while the solvent is considered as a structureless continuum. Such models are referred to as "primitive", while a full inclusion of all species including the solvent defines a "civilized" model (this terminology is attributed to R. H. Stokes [21]). Both the primitive [22-28] and civilized [20, 29-36] models are applied to study charged interfaces. Clearly, the civilized models are superior because they account for all species in the electrolyte solution. In fact, it was demonstrated that the primitive statistical mechanical models yield results that are identical to those obtained from solving the modified PBandelman equation [37]. Hence a truly realistic description of charged interfaces 
that involve electrolytes requires a civilized model that explicitly acknowledges the explicit presence of the solvent molecules alongside all ionic species.

Another important aspect of EDLs is associated with the mechanism of surface charge formation. It usually originates from dissociation of surface groups, ionic adsorption from the solution bulk or both [38]. These details are lost when Eq. (1) is solved as Dirichlet or a Neumann boundary value problem where either the surface potential $\Psi_{s}$ or the surface charge $\sigma=-\varepsilon \varepsilon_{0}(\nabla \Psi)_{s}$ are fixed at a constant value. This is not physically correct as pointed out by Ninham and Parsegian [39] who insisted that the boundary condition at the interface should reflect the thermodynamic equilibrium between the surface reactive groups and certain ionic species in the bulk referred to as potential determining ions (PDIs). This boundary condition is known as surface charge regulation (CR) and it presents a significant improvement in analyzing a variety of physical problems involving EDLs [40-49].

Until recently, the CR condition was not applied in conjunction with more elaborate statistical mechanical analysis of EDLs as those discussed above. An important step in that direction was taken by Heinen et al. [28], who applied the CR concept to a charged colloidal dispersion analyzed using the primitive (solvent excluded) OZ integral equation approach. The solvent effect in combination with CR boundary condition was first analyzed in our recent publication [36]. The analysis revealed that the surface CR condition strongly couples to the detailed solution structure in the immediate vicinity of the charged interface. This structure is dominated by the solvent because of its enormous concentration in comparison to that of the dissolved ions. The solvent molecules provide a structural matrix which determines the local ionic distribution. We have demonstrated that due the solvent effect the density distribution for the ionic species is liquid-like despite the fact that their actual concentration is gas-like [36].

In the present work, we perform an extensive analysis of the solvent impact on the properties of EDLs with surface charge regulation. Our particular focus is mostly on effects that have a non-electrostatic origin. These effects are largely responsible for experimental observations as the Hofmeister series $[50,51]$, the well-known electrokinetic measurements of Tadros and Lyklema [52], or the more recent results on the interactions between surfaces [53-55].

The paper is organized as follows: the next section outlines the DFT model for a double layer with CR condition at the charged interface. Section 3 present the results and discussion and Section 4 summarizes the conclusion.

\section{Theory}

\subsection{Density Functional Theory for Charged Interfaces}

The focus of our study is on charged interfaces that involve electrolyte solutions (see Fig. 1). Our model is civilized as it explicitly includes all solution components including the solvent. The charge and potential are dependent on the local distribution of all ionic species and the solvent clearly has an impact on it. Following the historical tradition we will refer to the physical interface between the charged interface and the electrolyte solution as the inner Helmholtz plane (IHP), the layer of ions and solvent molecules in the immediate vicinity to the charged interface as Stern layer, [5]. The midplane of the Stern layer defines the outer Helmholtz plane (OHP). There is another plane of interest that is located between the first layer of ions (and solvent molecules) in the immediate vicinity of the interface and the bulk solution. It is often assumed that this is the shear plane (SP) known from the theory of electrokinetic phenomena [38, 56-58]. Detailed molecular dynamics simulations have shown that indeed the stagnant layer thickness is usually of the order of ions or solvent molecular diameter [5963]. Hence, an electrokinetic measurement [57] is relevant to the charge and potential at the SP, after the first layer of ions and solvent molecules near the wall.

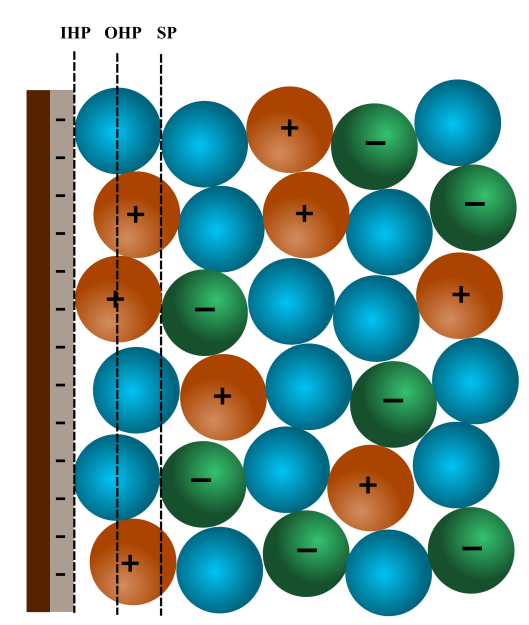

Fig. 1. A sketch of electrolyte solution in contact with a charged surface. The line on the right of the negative charges represents the inner Helmholtz plane (IHP). The dashed lines depicts the outer Helmholtz plane (OHP) and the dotted line illustrates the plane of electrokinetic charge, or the shear plane (SP).

The theoretical analysis is performed in the framework of a classical DFT [15, 16, 18, 20, 33, 36]. DFT is very well-suited to be applied to inhomogeneous fluid in an external field, and hence accurately describes an EDL. The electrolyte solution is represented by PDIs, non-PDIs and counterions to both. All are dissolved in a Lennard-Jones [14] type of solvent. The functional has the form

$$
\begin{gathered}
\Omega\left[\left\{\rho_{i}(z)\right\}\right]=k_{B} T \sum_{i=1}^{N} \int d z \rho_{i}(z)\left\{\ln \left[\lambda_{i}^{3} \rho_{i}(z)\right]-1\right\}+ \\
F_{H S}^{e x}\left[\left\{\rho_{i}(z)\right\}\right]+F_{\text {long }}^{\text {ex }}\left[\left\{\rho_{i}(z)\right\}\right]+ \\
\sum_{i=1}^{N} \int d z \rho_{i}(z)\left[V_{i}^{\text {ext }}(z)-\mu_{i}\right],
\end{gathered}
$$

where $\lambda=\sqrt{h^{2} /\left(2 \pi m_{i} k_{B} T\right)}$ is the thermal de Broglie wavelength, $h$ is the Planck's constant, $m_{i}$ is the mass of species " $i$ ", and $\rho_{i}(z)$ is the local density of component " $i$ ". The latter is a function of $z$, which is the coordinate normal to the interface. $V_{i}^{e x t}(z)$ is the external potential energy for species " $i$ " due to both electrostatic and $\mathrm{LJ}$ contributions, and $\mu_{i}$ are the chemical potentials of all species. $N$ is the total number of components: ions and solvent. The hard sphere (HS) interactions are 
accounted following the theory of Rosenfeld [19]. The longrange interactions have a hard-core Lennard-Jones (LJ) component

$$
\Phi_{\mathrm{LJ}}\left(r_{i j}\right)=4 \epsilon_{i j}\left[\left(\frac{d_{i j}}{r_{i j}}\right)^{12}-\left(\frac{d_{i j}}{r_{i j}}\right)^{6}\right], r_{i j}>d_{i j}
$$

where $d_{i j}=\left(d_{i}+d_{j}\right) / 2, d_{i}$ being the diameter of component " $i$ ". The LJ interaction of a molecule (or ion) of type " $i$ " with a wall is

$$
\Phi_{\mathrm{LJ}}(z)=\epsilon_{i}\left[\frac{2}{15}\left(\frac{d_{i}}{z}\right)^{9}-\left(\frac{d_{i}}{z}\right)^{3}\right], z>d_{i} / 2
$$

where $\epsilon_{i j}$ and $\epsilon_{i}$ determine the magnitudes of the LJ energy.

The charged species also exhibit Coulombic electrostatic (el) interactions (in addition to the $\mathrm{LJ}$ ) in the bulk

$$
\Phi_{\mathrm{el}}\left(r_{i j}\right)=\frac{q_{i} q_{j} e^{2}}{4 \pi \varepsilon \varepsilon_{0} r_{i j}}, r_{i j}>d_{i j},
$$

as well as with the charged wall

$$
\Phi_{\mathrm{el}}(z)=\frac{q_{i} \sigma z}{2 \varepsilon \varepsilon_{0}}, z>d_{i} / 2
$$

Where $\varepsilon$ and $\varepsilon_{0}$ are relative dielectric permittivity of the solvent, the dielectric constant of vacuum, respectively, and $\sigma$ represents the surface charge. The grand thermodynamic potential is then minimized with respect to the density distribution of all species according to

$$
\frac{\delta \Omega\left[\rho_{i}(z)\right]}{\delta \rho_{i}(z)}=0
$$

subject to the CR regulation condition (see section 2.2 below). Knowing the density distribution of each species (ions and solvent), one may compute the surface charge, which is equal to the excess bulk charge with an opposite sign

$$
\sigma=-\sum_{i=1}^{M} \int_{0}^{\infty} q_{i} \rho_{i}(z) d z
$$

where $M$ is the total number of components in the solution. The solvent is neutral, hence it is formally a component with zero charge $\left(q_{s}=0\right)$.

The minimization of the grand potential [see (13)] is performed using the Tramonto software [64], which was extended to account the charge regulation boundary condition (see next section 2.2).

This approach allows to determine the charge in any plane in the EDL that is parallel to the charged interface. This is important because different experiments lead to different result for the charge that is attributed to the interface. For example, surface titration provides the number of ionized groups that regulate the charge [65-68]. An alternative and very common experiment is based on analyzing electrokinetic motion (electrophoresis or electro-osmosis) [56-58]. This measurement provides information about the charge and potential after the Stern layer (see Fig. 1). Thus the titration method is applied to the charge at the IHP, while the electrokinetic experiments are more relevant to the charge at the SP. This leads to some ambiguity regarding the definition of PDIs. To avoid confusion in the present analysis, we define the PDIs as the species that are involved in a chemical exchange of charge such as in Eq. (9). All other charged species will be considered as non-PDIs even if they still interact with the interface via Coulombic or LJ forces. We discuss this issue in more detail in section 3 below.

Using a LJ model for the attractive interactions [see Eqs. (3) and (4)] is a simplification, which does not take into account any anisotropic dipole interactions that are present in polar liquids, water being the typical example. This means that a molecular interpretation of the solvent dielectric permittivity is not possible. Still, the Coulombic energies (5) and (6) need to be scaled down to realistic magnitudes. To achieve that, we use the bulk dielectric permittivity value for the solvent. This is an approximation, and if the dipole nature of the solvent molecules is properly taken into account the dielectric constant can be derived from molecular arguments [21, 29, 30, 34]. Still, for moderately concentrated solutions, such an approximation is quite reasonable $[21,30,69]$. The reason is that for not too concentrated electrolyte solutions, the average distance between two ions is large so that any perturbation in the solvent structure due to the presence of an ions relaxes to the bulk solvent state before reaching a second ion. For example for electrolyte concentration equal to $0.01 \mathrm{M}$, there are on the average about 35 solvent molecules between any two ions. The local polarization due to the presence of a charge drops within a few solvent diameters [21]. Hence 35 solvent molecules is a large enough number to safely assume that at room temperature each ion is surrounded by bulk dielectric.

In summary, our analysis is limited to hard-core, $\mathrm{LJ}$, and Coulombic interactions between the components and/or with the charged surface, but it captures the most important features of real electrolytes and allows one to study both electrostatic and non-electrostatic driven effects such as solvation interactions, specific interactions between species and with the interface, etc.

\subsection{Charge Regulation at the Surface Boundary of an Electric Double Layer}

The chemistry responsible for the charging of surfaces in contact with electrolyte solutions can be very complex involving a multitude of possible reactions [40]. We use a relatively simple yet sufficiently comprehensive model suggested by Chan et al. [43], which involves two surface reactions

$$
\begin{aligned}
\mathrm{AH}_{2}^{+}+\mathrm{BH} & \rightleftharpoons \mathrm{AH}+\mathrm{BH}_{2}^{+}, & & \mathrm{pK}_{+}=-\log _{10} \mathrm{~K}_{+} \\
\mathrm{AH}+\mathrm{BH} & \rightleftharpoons \mathrm{A}^{-}+\mathrm{BH}_{2}^{+}, & & \mathrm{pK}_{-}=-\log _{10} \mathrm{~K}_{-}
\end{aligned}
$$

where $\mathrm{K}_{+}$and $\mathrm{K}_{-}$are the equilibrium constants, and $\mathrm{AH}$ is a surface chemical group that can either bind or release a proton 
depending on local densities $\rho_{\mathrm{BH}_{2}^{+}}, \rho_{\mathrm{BH}}$ of species $\mathrm{BH}_{2}^{+}$and $\mathrm{BH}$ in the solution. The species $\mathrm{BH}$ can split according to the bulk reaction

$$
2 \mathrm{BH} \rightleftharpoons \mathrm{B}^{-}+\mathrm{BH}_{2}^{+}
$$

and produce a pair negative and positive ions. The positive species $\mathrm{BH}_{2}^{+}$is the PDI and it is its subsurface layer concentration that is involved in the equilibrium (9). The negative ions $\mathrm{B}^{-}$are natural counterions of the PDIs. In addition, there are also negative ions that are introduced as counterions of the nonPDIs (i.e., the background electrolyte). They do not participate in the reaction (10) but are otherwise identical in terms of size, charge and all $\mathrm{LJ}$ interactions to $\mathrm{B}^{-}$. Therefore, in the discussion below we will not discriminate between the types of negative ions that are present in the solution and will consider them as single species.

A proper account for the concentration of species in the subsurface (i.e. Stern) layer is very important. It determines the local concentration of each component and in the case of PDIs has an effect on the equilbria (9), and on the surface charge at the IHP. Since all solution species have finite size, we determine this concentration using \{see Supplemental Material in Ref. [36]\}

$$
\rho_{i}^{s}=\frac{\int_{0}^{d_{i} / 2} d z \rho_{\mathrm{i}}(z)}{\int_{0}^{d_{i} / 2} d z} .
$$

The surface charge then reads [43]

$$
\begin{aligned}
\sigma & =e \Gamma \frac{\rho_{\mathrm{AH}_{2}^{+}}^{s}-\rho_{\mathrm{A}^{-}}^{s}}{\rho_{\mathrm{AH}}^{s}+\rho_{\mathrm{AH}_{2}^{+}}^{s}+\rho_{\mathrm{A}^{-}}^{s}} \\
& =e \Gamma \frac{\delta \sinh \left[e\left(\Psi_{N}-\Psi_{s}\right) / k_{B} T\right]}{1+\delta \cosh \left[e\left(\Psi_{N}-\Psi_{s}\right) / k_{B} T\right]} .
\end{aligned}
$$

where $\delta=2 \sqrt{K_{-} / K_{+}}, \Gamma$ is the surface concentration of ionizable groups, and $\Psi_{s}$ is the surface potential. The quantity $\Psi_{N}=\left[\ln (10) k_{B} T / e\right]\left(\mathrm{pI}-\mathrm{pH}_{b}\right)$ is the Nernst potential where $\mathrm{pI}=\left(\mathrm{pK}_{-}+\mathrm{pK}_{+}\right) / 2$ is the surface isoelectric point and $\mathrm{pH}_{b}$ is the $\mathrm{pH}$ value in the bulk solution, far from the charged interface. The relationship (12) corresponds to a measurement of the surface charge using the titration method mentioned above.

The approach outlined in Section 2 allows for a description of an EDL in molecular details while taking into account the surface chemistry that governs the surface charge.

\section{Results and Discussion}

\subsection{Solution Structure and Its Effect on the Surface Charge}

The precise structure of an electrolyte solution strongly couples to the surface chemistry [see Eq. (9)], and therefore plays an important role in determining the surface charge [36]. Here we expand the analysis and present data that provide further insights into the physics underlying this observation. The effect of the solvent molecular structure can clearly be demonstrated by examining the dependence of the surface charge on the size of the solvent molecules. Varying that size from zero to a finite value (e.g., same as the size of the ions in the solution) is equivalent to gradually "turning on" the solvent contribution to the structure with all the excluded volume and attractive interactions that follow from it. We present two cases that correspond to (i) $\mathrm{pI}=2, \Delta \mathrm{pK}=8$, and (ii) $\mathrm{pI}=8, \Delta \mathrm{pK}=4$, where $\Delta \mathrm{pK}=\mathrm{pK}_{-}-\mathrm{pK}_{+}$(see Fig. 2). The number of surface ionizable groups for both cases is assumed to be $\Gamma=8 \times 10^{18} \mathrm{~m}^{-2}$. If fully ionized, this number leads to a dimensionless surface charge density $\sigma d^{2} / e= \pm 0.664$. The length-scale $d$ corresponds to a characteristic molecular (or ionic) diameter. It is set to $d=0.288 \mathrm{~nm}$, which ensures that for a reduced liquid density $\rho d^{3}=0.8$, the molarity is $55.56 \mathrm{M}$.

All above parameters were selected because they are within the typical range for common oxides like $\mathrm{SiO}_{2}$ and $\mathrm{Al}_{2} \mathrm{O}_{3}$ respectively [70]. The bulk $\mathrm{pH}=4$ for the solution in contact with $\mathrm{SiO}_{2}$ and $\mathrm{pH}=5$ for $\mathrm{Al}_{2} \mathrm{O}_{3}$. These were selected in order to provide examples for negative and positive surfaces, while being relevant to realistic situations.
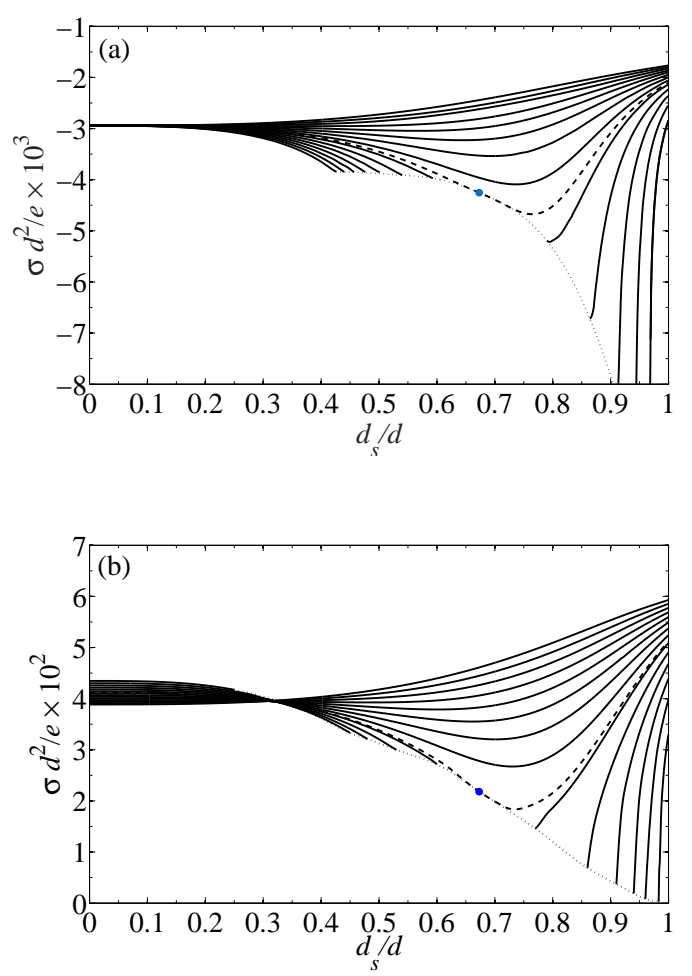

Fig. 2. Surface charge of an EDL as a function of the solvent molecular diameter. The different curves are for different values of the LJ parameter $\epsilon_{i j}$, which starts at zero and increases by increments of $0.1 k_{B} T$, with the exception of the dashed curve, which is for $\epsilon_{i j} / k_{B} T=0.76$. As $\epsilon_{i j}$ increases, the curves exhibit a transition from monotonic to non-monotonic behavior, and finally break at the spinodal (dotted curve). The dot corresponds to the critical point. (a) $\mathrm{pI}=2$, $\Delta \mathrm{pK}=8$ and $\mathrm{pH}=4$. (b) $\mathrm{pI}=8, \Delta \mathrm{pK}=4$ and $\mathrm{pH}=5$.

Fig. 2 demonstrates the effect of the solvent structure and LJ attraction on the resultant dimensionless surface charge $\sigma d^{2} / e$, according to the chemical equilibrium (9). Only bulk LJ interactions are included. The LJ interactions of all solvent component with the wall are considered to be zero. The wall however 
is hard and impenetrable and its surface charge generates an electric potential that affects the distributions of all ions in the EDL. Fig. 2a corresponds to $\mathrm{pI}=2, \Delta \mathrm{pK}=8$ and $\mathrm{pH}=4$ and Fig. $2 \mathrm{~b}$ to $\mathrm{pI}=8, \Delta \mathrm{pK}=4$ and $\mathrm{pH}=5$. The solution ionic strength (including all ionic contributions) is equal to $0.01 \mathrm{M}$. As the attraction becomes stronger (in steps of $\Delta \epsilon_{i j} / k_{B} T=0.1$ ), the trend of increasing the surface charge $\sigma$ with $d_{s} / d$ becomes weaker and non-monotonic. The surface charge dependence on the solvent diameter is very strong when the latter approaches those of the ions $\left(d_{s} / d \rightarrow 1\right)$. This means that small variations in the relative dimensions may have a significant effect on the surface charge. The surface charge depends on the equilibrium between the surface reactive groups and the PDIs in the stern layer. The repulsive interactions involving the PDIs in the bulk will shift the equilibrium in favor of reducing the surface charge magnitude because the free energy will drop as the ions move from the bulk towards the interface. On the other hand, increasing the attractive bulk interactions with the solvent (e.g., solvation) will have the opposite effect on the free energy and thus will favor an increase in the surface charge.

The increase of the solvent diameter in Fig. 2 is formally equivalent to increasing the liquid density, and in presence of attractions this lead to liquid-vapor phase separation, which is indicated in the figures by the dashed lines. They correspond to the spinodal lines and depict the domains of thermodynamic instability. The behavior at $d_{s} \rightarrow 0$ is not realistic because it also corresponds to a systems of very low gas-like density. The more realistic civilized analysis corresponds to the far right side of the figure. Clearly any analysis that ignores the solvent correlation misses a number of physical effects and is therefore incomplete. However, a proper account of the solvent molecular structure alone is not sufficient if the surface charge regulation condition (9) is replaced by constant surface potential or constant surface charge boundary condition. This wide variety of physical effects is due to the coupling between the surface charge regulation and the complete solution structure. Examining Fig. 2, it is clear that using a primitive electrolyte model to describe EDLs suffers similar shortcomings as the application of the ideal gas equation of state to dense liquids.

The cases depicted in Figs $2 \mathrm{a}$ and $2 \mathrm{~b}$ are similar in some aspects and quite different in others. The common features were outlined above and here we discuss the differences. Fig. $2 \mathrm{a}$ is for a relatively weakly negatively charged surface, while Fig. $2 \mathrm{~b}$ is for a positive surface and the charge magnitude is greater. This leads to a noticeably different behavior near the limit of vanishing solvent molecular size, which is the domain of the primitive model. As the solvent diameter goes to zero all curves in Fig. 2a collapse onto the same value for the surface charge. In Fig. $2 b$ the curves intercept the vertical axis at different values for the surface charge. The reason for that is because the higher surface charge magnitude (in comparison with Fig. 2a) attracts a greater number of counterions (negative in this case) and the inter-ionic correlations are present. We remind that in the primitive model, the solvent correlations are not taken into account. However, all ions participate in the model with their physical size and interactions. The absence of explicit solvent in the model leads to an overestimate of the ionic local concen-

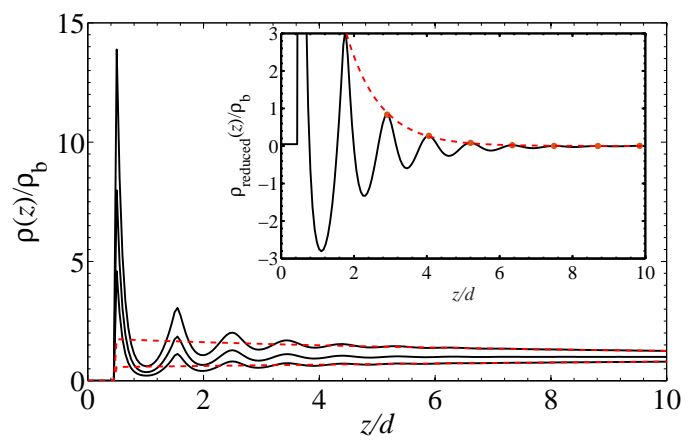

Fig. 3. Density distribution for ions and solvent in the vicinity of a negatively charged interface $\mathrm{pI}=2, \Delta \mathrm{pK}=8$ and $\mathrm{pH}=4$, in absence of attractive LJ interactions. The top curve is for the PDIs and positive non-PDIs, which follow exactly the same trend. The middle curve corresponds to the density of the solvent molecules and the bottom curve is for the negative ions. The smooth dashed curves depict the primitive model solution. The inset shows the structural effect. Peak height exhibits an exponential decay - see the text for more details.

trations and correlations in the vicinity of the charged interface. These correlations are dependent on the LJ interactions, which leads to the variations in the surface charge observed in the limit of $d_{s} \rightarrow 0$. Interestingly, as the solvent structural contribution increases, it seems to offset the ion-ion correlation induced variations and completely eliminate them around $d_{s} / d \simeq 0.3$. After that point the curves for various LJ parameters $\epsilon_{i j}$ diverge from one another again as $d_{s} / d \rightarrow 1$. Another important difference between the two figures is that the critical point in Fig. 2b is shifted towards slightly higher attractive LJ en$\operatorname{ergy}\left(\epsilon_{i j} / k_{B} T \simeq 0.8\right.$ instead of 0.76$)$. This observation can also be attributed to the greater ionic concentration in the EDL due to the more significant surface charge magnitude (compared to the case in Fig. 2a). Most of the ions in the EDL have the same charge (opposite to that of the interface) and hence repel each other. As a result, it requires more attractive energy to bring them closer into a liquid state.

In our earlier work [36], we have found that there are two characteristic lengths associated with the EDLs. One is the well known Debye wavelength [71]

$$
\kappa=\left(\frac{1}{\varepsilon \varepsilon_{0} k_{B} T} \sum_{i} \rho_{i}^{0} q_{i}^{2}\right)^{1 / 2}
$$

where $\rho_{i}^{0}$ is the bulk concentration of species $i$. Fig. 3 presents data for the density distribution of all species in the vicinity of the charged interface. The first and highest peak corresponds to the Stern layer, while the minimum after it defines the location of the SP (see Fig. 1). A second characteristic length is a measure of the structural effects and their decay with the distance from the charge surface. Following the work of Martynov [72], we analyzed the peak heights of the local density distribution after subtracting all long-range contributions such as Coulombic or LJ interactions (see Fig. 3). The reduced density then is defined as $\rho_{\text {reduced }}=\rho_{\mathrm{DFT}}-\rho_{\mathrm{PB}}$, where the subscript DFT and $\mathrm{PB}$ refer to a civilized density functional and continuum 


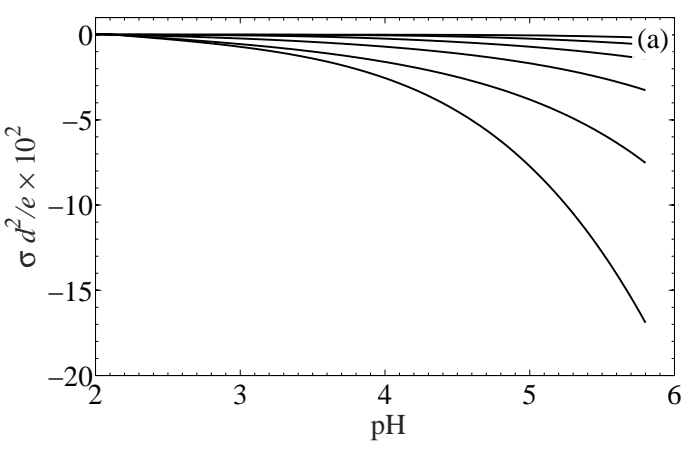
suming that all species have the same size) [36].

\subsection{Effects of the Surface Chemistry.}

a higher value, which is due to the effect of the finite size of all participants. In the charge regulation model that we use, the equilibrium constants [see Eq. (9)] do not explicitly account for the any effects due to the molecular size of the solution species. This effects are added with the implementation of the civilized DFT approach and lead to the observed shift.

\subsection{Effects of the Bulk Solvation Interactions.}

Interactions in the bulk of the solution compete with those with the surface. Since the solvent molecules are in overwhelming number, their interaction with the ionic species or among themselves will dominate. The importance of such analysis follows from the experimental fact that ionic solvation varies substantially from one species to another. For example, moving down the alkali column in the periodic table shows that the ions become less prone to water solvation [73].

The surface charges in the present analysis are obtained at two locations: the physical interface (IHP) and after the first layer of ions, i.e. the SP (see Fig. 5). We examine the effect of the solvent LJ interactions [i.e., the parameter $\epsilon_{i j}$ - see Eq. (3)] with each component: solvent-PDIs (solid curves), solventpositive non-PDIs (dashed curves), solvent-negative ions (dotted curves) and solvent-solvent (dashed-dotted curves). Each curve was calculated while all other LJ interactions were set to $\epsilon_{i j} / k_{B} T=1$. This is the reason why all curves intersect at that point. The remaining parameters are the same as in Fig. 2a.

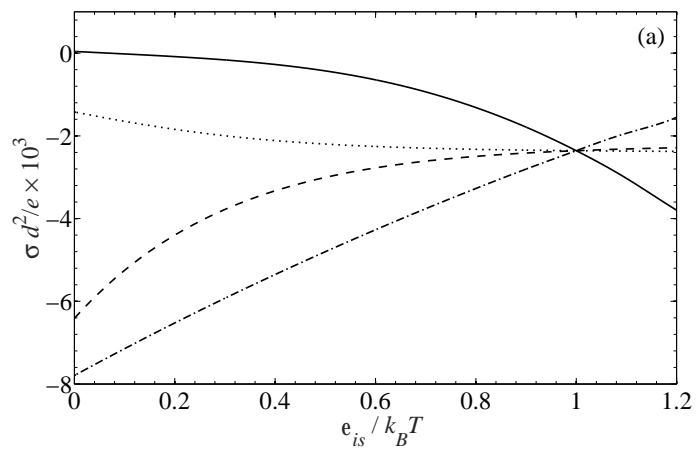

Poisson-Boltzmann solution respectively. In this case, all density distribution curves collapse onto a single one and its peak heights values decrease exponentially, i.e. $\sim \exp (-\beta z)$. The wavelength associated with the decrease is $\beta=1.3 d$ (as-

The charge at the interface between the electrolyte solution and the other phase (i.e., the IHP) is governed by the reaction equilibria (9), which in turn is characterized by the equilibrium constants, or equivalently by the values of $\mathrm{pK}_{+}$and $\mathrm{pK}_{-}$. These parameters determine how the surface groups will respond to the local concentration of the PDIs. The latter is determined by the value of the pH. Fig. 4 shows the results for two different EDL systems. Fig. 4a corresponds to a surface isoelectric point $\mathrm{pI}=2$ and Fig. $4 \mathrm{~b}$ to $\mathrm{pI}=5$. The total concentration of electrolyte is $0.01 \mathrm{M}$, therefore the lower limit on the horizontal axis is $\mathrm{pH}=2$. The different curves in both panels reflect the specific surface chemistry in terms of the value of the parameter $\Delta \mathrm{pK}=2,4,6,8,10$, and 12 . The smaller $\Delta \mathrm{pK}$ the more responsive the surface is to $\mathrm{pH}$ variations (see also Ref. [46]). Hence, the magnitude of the surface charge depends on the surface $\mathrm{pI}$, the solution $\mathrm{pH}$ and the parameter $\Delta \mathrm{pK}$. Larger differences between the $\mathrm{pI}$ and $\mathrm{pH}$, lead to greater magnitudes of surface charge. The overall behavior of charge regulating surfaces described above, is qualitatively consistent with experimental observations (see for example Ref. [52]). The actual isoelectric point for $\mathrm{pI}=5$ (see Fig. $4 \mathrm{~b}$ ) is slightly shifted to

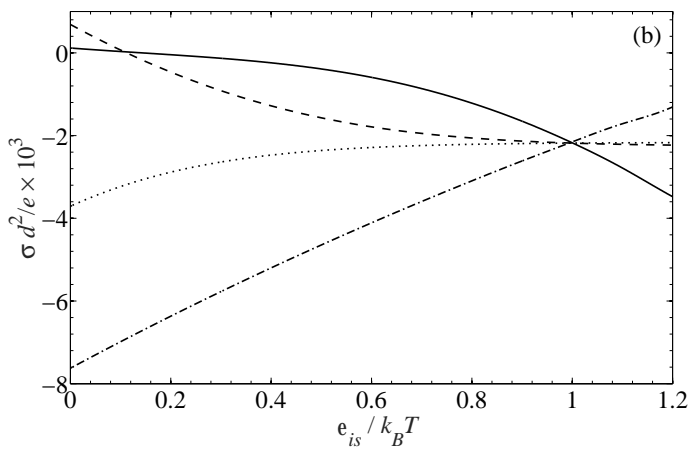

Fig. 5. Solvation effects on charge at the actual interface(a) and at the shear plane(b). The curves represent charge variation with PDIs-solvent (solid curves), Non-PDI positive ions - solvent (dashed curves), solvent-solvent (dotdashed curves) and negative ions-solvent (dotted curves) interaction energies. 
Fig. 5a shows the effect of the bulk solvation interactions for all species on the charge at the IHP. If the PDIs (solid curve) are not too solvated they accumulate near the surface and neutralize the negative charges according to the reaction (9). However, as the solvent-PDI attraction increases the PDIs move away from the surface in the bulk, which naturally increases, the negative charge at the interface. The effect of the PDIs on the charge at the SP seems to follow the same trend as the one at the IHP (see the full curve in Fig. 5b). As the PDIs get more solvated the electrokinetic charge at the SP becomes more negative.

The solvation of the positive non-PDIs has a strong impact on the surface charge at the IHP (see the dashed curve in Fig. 5a). The charge magnitude at the IHP decreases with the increase of the solvation of the positive non-PDIs. Low solvation of the positive non-PDIs leads to increased accumulation near the interface, which in turn creates a repulsive electrostatic barrier for the positive PDIs to approach the interface thus reducing their concentration in the Stern layer, As a result the equilibrium (9) shifts towards a larger number of negatively charged surface groups $\mathrm{A}^{-}$. Increasing the solvation interactions favors a reduction of the concentration of non-PDIs in Stern layer and thus allows for a greater number of PDIs to reach the charged surface and neutralize some of the charged surface groups.

The effect of the non-PDIs on the charge at the SP however, is very different. If the non-PDIs are not (or very weakly) solvated they tend to accumulate near the surface in the Stern layer. The latter becomes predominantly positive, which leads to an overall positive charge at the SP. As the solvation of the non-PDIs becomes stronger, they tend to leave the Stern layer, which results in more negative charge at the SP.

The solvent-solvent interaction seems to have a very similar effect on the charge both at the IHP (see the dot-dashed line in Fig. 5a) and the SP (dot-dashed line in Fig. 5b). A low LJ interaction between the solvent molecules leads to their preferential attraction to the rest of the species (including the PDIs, and non-PDIs) and facilitates their removal from the surface. As a result the local concentration of the positive PDIs and nonPDIs near the charged interface is low and the surface carries a highly negative charge. As the attraction between the solvent molecules increases, they will tend to maximize contacts with each other and minimize those with the PDIs. Hence, the PDI concentration near the charged interface decreases and the negative charge is reduced according the surface equilibria (9). Similarly, the non-PDIs numbers near the interface is reduced leading to their lower concentration in the Stern layer, which in turn determines the charge at the SP.

The solvation of the negative ions also affects the charges at the IHP and SP. The magnitude of the negative surface charge at the IHP (see the dotted line in Fig. 5a) slightly increases with the solvation of the negative ions. The reason for that effect is also in the electrostatic interactions with the PDIs. Larger numbers of negative ions near the surface attract the positive PDIs, thus reducing the surface charge according to Eq. (9). The charge at the SP on the other hand depends on the balance between the charges at the IHP and those in the Stern layer. Fig. $5 \mathrm{~b}$ shows that the SP charge becomes less negative as the negative ions become more solvated. This is due to the reduction of the negative charges in the Stern layer, and hence results in lower overall negative charge at the SP.

The solvation interactions, while non-electrostatic in nature, have a very strong effect on the properties of charged interfaces that involve electrolytes. Hence, they allow to qualitatively explain why ionic species with same charge numbers may lead to different surface charge, or why different experiments (e.g., surface charge titration and electrokinetic measurements) may lead to different results.

\subsection{Effects of the Non-Electrostatic Solution-Surface Interac- tions.}

All solution species experience various interactions with the surface. It is obvious that the ions exhibit electrostatic interactions with the charged interface. However, the focus in this subsection is on non-electrostatic forces of LJ type [see (4)]. These interactions in combination with the solvation (see the previous subsection) determine all effects that are unrelated to charges but known to exist and affect the surface charge and the overall properties of EDLs [50-52, 73].

Fig. 6 presents DFT results for the surface charge vs the LJ interaction energy with the interface for each component. The surface is characterized by $\mathrm{pI}=2, \Delta \mathrm{pK}=8$ and it is in contact with solution that has $\mathrm{pH}=4$. The data in Figs. $6 \mathrm{a}$ and $6 \mathrm{~b}$ shows the charge at the IHP, while Figs. $6 \mathrm{c}$ and $6 \mathrm{~d}$ refer to the charge at the SP after the Stern layer. Figs. 6a and $6 \mathrm{c}$ cover a wider range of species-wall interaction energies. Figs. $6 \mathrm{~b}$ and $6 \mathrm{~d}$ show the details at moderate surface attraction, which are rather complex.

Both the IHP and SP charge values suggest that the effect of the surface interaction for the PDIs and the solvent are similar. As the PDIs get more attracted to the surface, their local concentration increases and the equilibrium (9) shifts towards lower number of the negative $\mathrm{A}^{-}$, and higher number of neutral $\mathrm{AH}$, or even positive $\mathrm{A}_{2}^{+}$surface groups. This effectively shifts the charge at the IHP and shear plane to less negative values. One important difference is observed for large values of $\epsilon_{\mathrm{PDI}} / k_{B} T$. The rate of change of the charge at the IHP tends to decrease, while it is almost constant at the SP. The reason is that the charge at the IHP depends on the chemical equilibrium (9), which determines how many of the surface groups turn from negative to neutral or positive. The charge at the SP however, is governed not only by the surface reaction, but also on the accumulation of positive PDIs in the Stern layer. The charge of these ions adds to the charge at the IHP, which leads to much stronger positive shift.

In contrast, the effects of the positive non-PDIs on the charges at the IHP and SP are very different. As the LJ attraction of the non-PDIs to the surface increases, the charge at the IHP starts decreasing and above $\epsilon_{\text {non-PDI }} / k_{B} T \simeq 5$ sharply drops down to $\sigma d^{2} / e=0.664$, which corresponds to complete dissociation of all surface groups [see Fig. 6a and Eq. (9)]. The reason for this strong effect follows from the fact as non-PDIs accumulate in great numbers near the charged surface, they create a excluded volume and electrostatic barrier for the PDIs to approach the surface and participate in the surface chemical reactions. Hence, the charge at the IHP increases to its maximum 

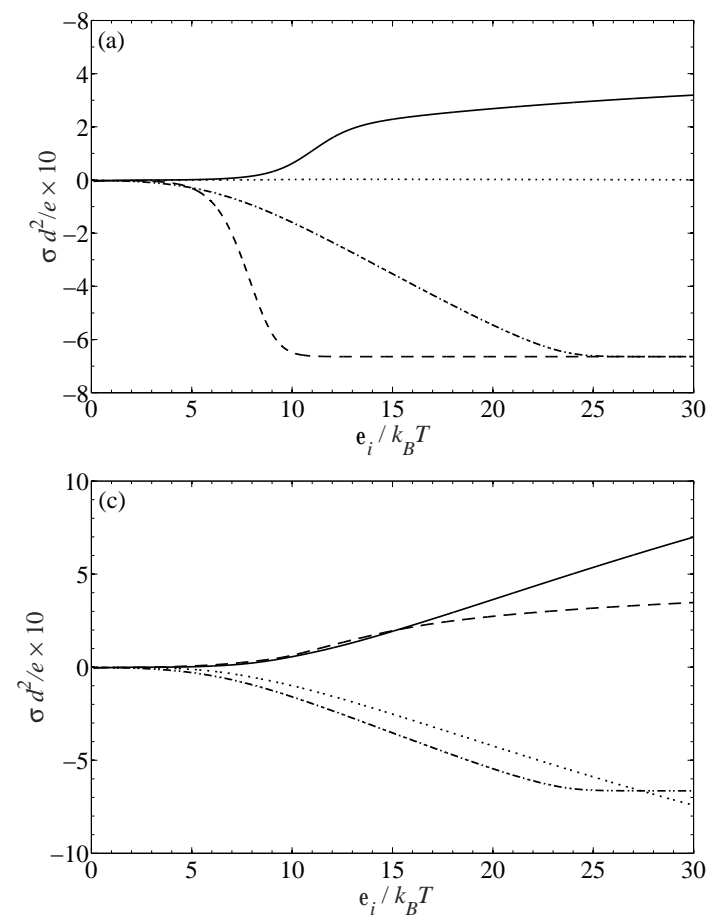
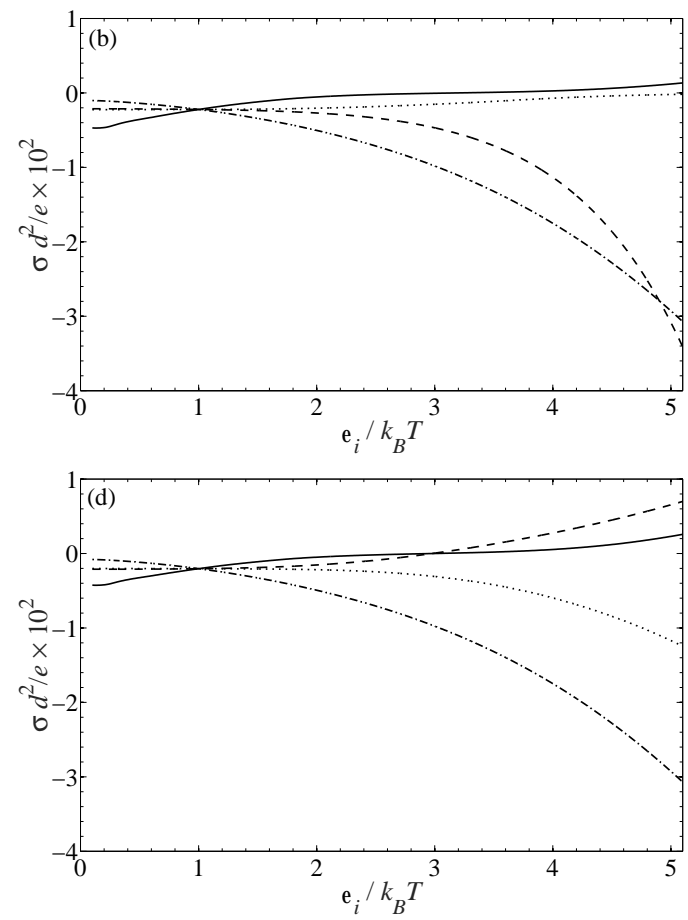

Fig. 6. Effect of LJ interactions with the wall for PDIs (solid curves), non-PDI positive ions (dashed curves), solvent (dot-dashed lines) and negative ions (dotted curves) on the surface charge. All other parameters are as in Fig. 2a. Panels (a) and (b) show the charge at the IHP. Panels (c) and (d) depict the charge at the SP. Panels (b) and (d) provide more detailed resolution of complex dependence of the surface charge on the LJ interactions for lower values of $\epsilon_{i} / k_{B} T$.

value. On the other hand the charge at the SP responds very differently to the increased attraction of the non-PDIs to surface (see Fig. 6b). In this case the surface charge becomes more positive, because of the accumulation of positive ions in the Stern layer. The observable charge at the SP is a difference between the negative surface charge at the IHP minus the positive ions in the Stern layer. Hence, as more positive ions populate the Stern layer, the more positive the SP charge becomes.

The LJ interaction of solvent molecules with the charged surface also has a strong effect both on the charge at the IHP and SP. This interaction determines the solvophilic/solvophobic properties of the material in contact with the electrolyte solution. As the surface becomes more solvophilic, the surface charge becomes more negative and above $\epsilon_{s} / k_{B} T \simeq 24$ reaches the fully dissociated state, where again $\sigma d^{2} / e= \pm 0.664$. This effect is entirely due to displacement of PDIs (as well as other ions) through simple excluded volume interactions. The dependencies on both Figs. 6a and $6 \mathrm{~b}$ look very similar. The reason for that is because the Stern layer is exclusively populated by uncharged solvent molecules and therefore there is practically no charge screening between the IHP and the SP. The charge on both sides of the Stern layer (see Fig. 1) is almost identical.

The effect of the negative ions present in the solution on the charge at the IHP is negligible (see Fig. 6a), and is significant on the charge at the SP (see Fig. 6c). The charge at the IHP is determined by the surface chemistry and the negative ions do not affect it much (see Fig. 6a). On one hand they are attracted to the surface and occupy volume, which should prevent PDIs from approaching the surface and participating in the equilibrium (9). The excluded volume effect however is offset by the electrostatic attraction that all positive ions experience and the overall effect is insignificant. The charge at the SP, however, becomes more negative as greater numbers of negative ions are attracted to the surface. The dependence on the attractive $\mathrm{LJ}$ energy becomes more pronounced with $\epsilon_{\text {negative ion }} / k_{B} T$ and for large values linearly decreases. It may be expected that at even higher energies the charge dependence may level off when the whole Stern layer is entirely populated by negative ions, add negative charge to the one already at the IHP.

\subsection{Effects of the Bulk Ionic Strength.}

The total bulk electrolyte concentration (i.e., ionic strength) modifies the potential distribution in the EDL through screening. Hence, it affects the potential at the surface, which in turn reflects on the surface charge [see Eq. (12)]. Figure 7 illustrates the effect of the total bulk electrolyte concentration in terms of ionic strength $I=\frac{1}{2} \sum_{i} z_{i}^{2} C_{i}$. The molar concentrations $C_{i}$ includes all ionic species, including the contributions of the PDIs, which depend on the solution $\mathrm{pH}$. The surface charge at three different $\mathrm{pH}$ values are analyzed. The $\mathrm{pH}$ effect is obvious and it is due to the chemical equilibria (9) [see also (12)]. The decrease of the surface charge with the bulk concentration of non-PDIs is a result of the overall charge and potential modification that stems from bulk charge screening. It modifies the overall potential distribution in the EDL as well as the surface potential $\Psi_{s}$ [see (12)].

Fig. $7 \mathrm{a}$ shows the charge at the IHP and Fig. $7 \mathrm{~b}$ corresponds to the charge at the SP. The values for the surface charges in these two cases are very different since the net charge of the 

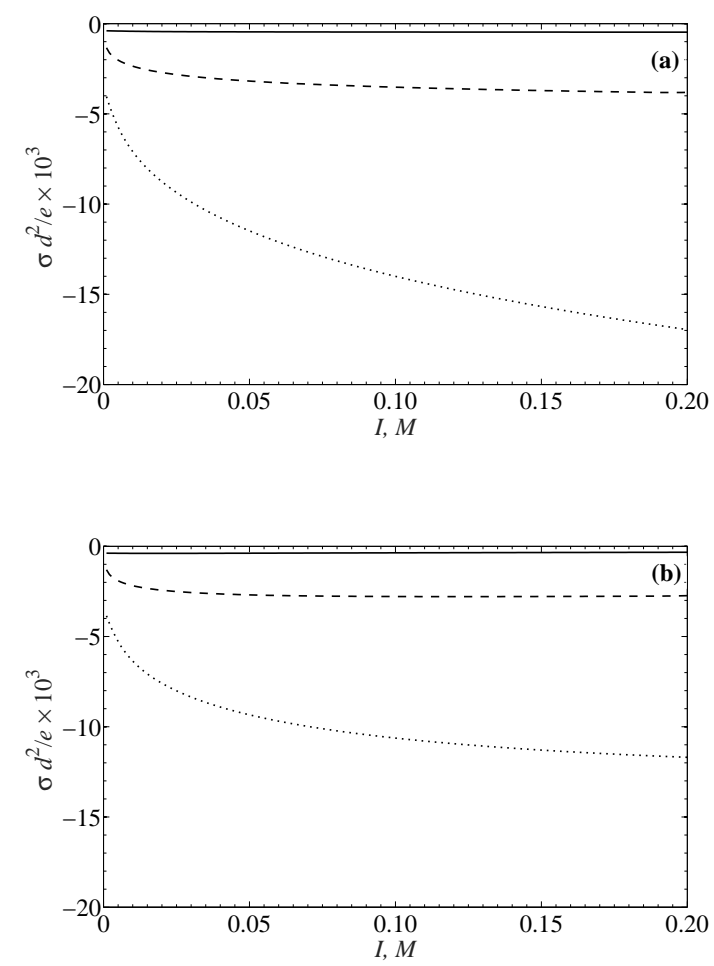

Fig. 7. Surface charge of an EDL as a function of the solution ionic strength in moles per liter. $\mathrm{pI}=2, \Delta \mathrm{pK}=8$. The three different cases are for $\mathrm{pH}=3$ (solid curves), $\mathrm{pH}=4$ (dashed curves), and $\mathrm{pH}=5$ (dotted curves). (a) Charge at the actual interface; (b) Charge at the outer boundary of the Stern layer.

Stern layer is effectively subtracted from the charge due to actual ionization of the surface. This is in general qualitative agreement with experimental observations [65-68] which predict a charge variation with the $\mathrm{pH}$ of the solution.

\section{Summary and Conclusions}

The charge formation at electrolyte interfaces and the properties of EDLs are complex functions of many parameters related to the material properties of the surface and the adjacent solution. The interfacial charge at the IHP is determined by surface chemical reactions (charge regulation) involving the surface reactive groups and components of the solution. All solution components contribute to the final surface equilibria, including species that are not directly participating in the surface reactions, (9). We show that the precise and detailed solution structure in the vicinity of the interface couples to the charge governing chemical equilibria. The solvent molecules play an important role in establishing this structure due to their exceedingly large number. The solvent contributes to the ionic structure through the excluded volume and LJ interactions. Hence, an accurate physical representation of charged interfaces involving electrolytes requires rigorous statistical mechanical approaches that explicitly account for all solution species and the correlations among them.

The surface chemistry plays an important role for the charge regulating behavior in EDLs. It depends on the material prop- erties of the interface and the composition of the electrolyte solution in contact with it.This is governed by three parameters: the pI of the surface, the bulk concentration of PDIs (in case of hydronium ions this is conveniently expressed by the $\mathrm{pH}$ ), and the difference $\Delta \mathrm{pK}=\mathrm{pK}_{-}-\mathrm{pK}_{+}$. The situation could be more complicated if more complex surface chemistry is present [40].

The analysis also asserts that the properties of an EDL with surface charge regulation strongly depends on non-Coulombic interactions and correlations in the electrolyte solution. Examples include the bulk solvation as well as non-electrostatic interactions with the EDL interface. The analysis of the solvation and surface interactions explains the fact that different types ions carrying the same charge may lead to a different outcome for the resultant surface charge. It also explains why components that are not directly involved in the surface chemical reactions affect the EDL properties. Hence, the combination of solvation and surface interactions can account for experimental observations that lead to the definition of the Hofmeister series[50, 51], electrokinetic results [52], or the ions-specific surfaces forces [53-55]

An important aspect of this study is to provide a framework for proper analysis of experimental data. As discussed above depending on the method one may directly probe the charge at the surface by counting the number of charged groups (using titration) or detect the charge at the shear plane (using electrokinetic methods). These could be and often are very different. Civilized DFT allows to distinguish between these cases and provides explanation for the difference based on rigorous statistical mechanical arguments.

The main focus of this study is on the properties of static EDLs. However, DFT can be generalized to non-equilibrium systems $[17,74]$. This will allow solving problems pertinent to surface reactions and solute diffusion.

In summary we believe that civilized statistical mechanical approaches (such as DFT) together with a proper account for all chemical equilibria at the EDL boundary offers the best theoretical background for modeling and analysis of charged interfaces that involve electrolyte solutions. The simple model presented in this work can be extended to include more components and/or more sophisticated functionals. This is a strategy that allows obtaining further insights into the properties of charged colloidal systems.

Acknowledgements : This research was supported by the Air Force Academy under cooperative agreement FA7000-142-0017 through funding from the Corrosion Policy and Oversight office, and the United States Department of Energy, Office of Basic Energy Sciences, Division of Materials Sciences and Engineering. We are thankful to the UNM Center for Advanced Research Computing for providing support and access to computational resources.

\section{References}

[1] H. Helmholtz, Ueber einige gesetze der vertheilung elektrischer strme in krperlichen leitern mit anwendung auf die thierisch-elektrischen versuche, Ann. Phys. Chem. 165 (1853) 211-233.

[2] G. Gouy, Sur la constitution de la charge electrique 'a la surface dun electrolyte, J. Physique 9 (1910) 457-468. 
[3] D. L. Chapman, A contribution to the theory of electrocapillarity, Phil. Mag. 25 (1913) 475-481.

[4] L. D. Landau, E. M. Lifshitz, Electrodynamics of Continuous Media, Nauka, 1982.

[5] O. Stern, Zur theorie der elektrolytischen doppelschicht, Z. Electrochem 30 (1924) 508-516.

[6] D. C. Graham, The electrical double layer and the theory of electrocapillarity, Chem. Rev. 41 (1947) 441-501.

[7] D. A. I. Borukhov, H. Orland, Steric effects in electrolytes: A modified poisson-boltzmann equation, Phys. Rev. Lett. 79 (1997) 435-438.

[8] D. H. D. Ben-Yaakov, D. Andelman, R. Podgornik, Beyond standard poisson-boltzmann theory: Ion-specific interactions in aqueous solutions, J. Phys.: Condens. Matter 21 (2009) 424106.

[9] R. P. D. Ben-Yaakov, D. Andelman, D. Harries, Ion-specific hydration effects: Extending the poisson-boltzmann theory, Curr. Opin. Colloid Interface Sci. 11 (2011) 542-550.

[10] M. v. d. V. P. M. Biesheuvel, W. Norde, A modified poisson-boltzmann model including charge regulation for the adsorption of ionizable polyelectrolytes to charged interfaces, applied to lysozyme adsorption on silica, J. Phys. Chem. B 109 (2005) 4172-4180.

[11] J. J. López-García, J. Horno, Poisson-Boltzmann description of the electrical double layer including ion size effects, Langmuir 27 (23) (2011) 13970-13974.

[12] R. Balescu, Equilibrium and non-equilibrium statistical mechanics, Wiley, New York, 1975.

[13] J. P. Hansen, I. R. McDonald, Theory of Simple Liquids, Academic Press, New York, 1976.

[14] D. A. McQuarrie, Statistical Mechanics, University Science Books, 2000

[15] R. Evans, Density functionals in the theory of nonuniform fluids, in: D. Henderson (Ed.), Fundamentals of Inhomogeneous Fluids, Marcel Dekker, Inc., New York, 1992, Ch. 3, pp. 85-175.

[16] J. Wu, Density functional theory for chemical engineering: From capillarity to soft materials, AIChE Journal 52 (3) (2006) 1169-1191.

[17] R. Evans, M. M. T. da Gama, Spinodal decomposition in a lennard-jones fluid, Mol. Phys. 38 (1979) 687-698.

[18] R. Roth, Fundamental measure theory for hard-sphere mixtures: a review, J. Phys.: Condens. Matter 22 (2010) 063102.

[19] Y. Rosenfeld, Free-energy model for the inhomogeneous hard-sphere fluid mixture and density functional theory of freezing, Phys. Rev. Lett. 63 (1989) 980-983.

[20] L. J. D. Frink, F. van Swol, Solvation forces and colloidal stability - a combined monte-carlo and density-functional theory approach, J. Chem. Phys. 100 (1994) 9106-9116.

[21] S. Carnie, D. Y. C. Chan, The structure of electrolytes at charged surfaces: Iondipole mixtures, J. Chem. Phys. 73 (1980) 2949-2957.

[22] M. Plischke, Pair correlation functions and density profiles in the primitive model of the electric double layer, J. Chem. Phys. 88 (1988) 27122718.

[23] Y. X. Yu, J. Z. Wu, G. H. Gao, Density-functional theory of spherical electric double layers and zeta potentials of colloidal particles in restrictedprimitive-model electrolyte solutions, J. Chem. Phys. 120 (2004) 72237233.

[24] Z. Tang, L. E. Striven, H. T. Davis, Interactions between primitive electrical double layers, J. Chem. Phys. 97 (1992) 9258-9266.

[25] O. Pizio, S. Sokolowski, On the effects of ion-wall chemical association on the electric double layer: A density functional approach for the restricted primitive model at a charged wall, J. Chem. Phys. 125 (2006) 024512 .

[26] J. Jiang, D. Cao, D. Henderson, J. Wu, Revisiting density functionals for the primitive model of electric double layers, J. Chem. Phys. 140 (2014) 044714.

[27] L. Blum, D. Henderson, Statistical mechanics of electrolytes at interfaces, in: D. Henderson (Ed.), Fundamentals of Inhomogeneous Fluids, Marcel Dekker, Inc., New York, 1992, Ch. 6, pp. 239-276.

[28] M. Heinen, T. Palberg, H. Löwen, Coupling between bulk- and surface chemistry in suspensions of charged colloids, J. Chem. Phys. 140 (2014) 124904.

[29] S. A. Adelman, J. M. Deutch, Exact solution of the mean spherical model for strong electrolytes in polar solvents, J. Chem. Phys. 60 (1974) 39353949.

[30] D. Y. C. Chan, D. J. Mitchell, B. W. Ninham, B. A. Pailthorpe, On the theory of dipolar fluids and iondipole mixtures, J. Chem. Phys. 69 (1978) 691-696.

[31] L. Blum, D. Henderson, Mixtures of hard ions and dipoles against a charged wall: The ornsteinzernike equation, some exact results, and the mean spherical approximation, J. Chem. Phys. 74 (1981) 1902-1910.

[32] W. Dong, M. L. Rosinberg, A. Perera, G. N. Patey, A theoretical study of the solidelectrolyte solution interface. i. structure of a hard sphere iondipole mixture near an uncharged hard wall, J. Chem. Phys. 89 (1988) 4994-5009.

[33] R. D. Groot, Density-functional theory for inhomogeneous fluids, Phys. Rev. A 37 (1988) 3456-3464.

[34] L. Perera, M. L. Berkowitz, Dynamics of ion solvation in a stockmayer fluid, J. Chem. Phys. 96 (1992) 3092-3101.

[35] A. M. J. W. Lee, J. A. Templeton, Atomistic and molecular effects in electric double layers at high surface charges, Langmuir 31 (2015) 74967502 .

[36] F. v. S. M. E. Fleharty, D. N. Petsev, Solvent role in the formation of electric double layers with surface charge regulation: A bystander or a key participant?, Phys. Rev. Lett. 116 (2016) 048301.

[37] P. Attard, Electrolytes and the electric double layer, Adv. Chem. Phys. 92 (1996) 1-159.

[38] S. S. Dukhin, Development of notions as to mechanism of electrokinetic phenomena and the structure of the colloid micelle, in: E. Matijevic (Ed.), Surface and Colloid Science, Wiley Interscience, New York, 1974, Ch. 1, pp. 1-49.

[39] B. W. Ninham, V. A. Parsegian, Electrostatic potential between surface bearing ionizable groups in ionnic equilibrium with physiologic saline solution, J. Theor. Biol. 31 (1971) 405-428.

[40] R. Ettelaie, R. Buscall, Electrical double layer interactions for spherical charge regulating colloidal particles, Adv. Coll. Interf. Sci. 61 (1995) 131-160.

[41] D. Y. C. Chan, T. W. Healy, T. Supasiti, S. Usui, Electrical double layer interactions between dissimilar oxide surfaces with charge regulation and stern-grahame layers, J. Colloid Interface Sci. 296 (2006) 150-158.

[42] S. H. Behrens, M. Borkovec, Electric double layer interaction of ionizable surfaces: Charge regulation for arbitrary potentials, J. Chem. Phys. 111 (1999) 382-385.

[43] D. Y. C. Chan, T. W. Healy, L. R. White, Electrical double layer interactions under regulation by surface ionization equilibria-dissimilar amphoteric surfaces, J. Chem. Soc., Faraday Trans. I 72 (1976) 2844-2865.

[44] S. H. Behrens, M. Borkovec, Electrostatic interaction of colloidal surfaces with variable charge, J. Phys. Chem. 103 (1999) 2918-2928.

[45] Z. Jiang, D. Stein, Charge regulation in nanopore ionic field-effect transistors, Phys. Rev. E. 83.

[46] M. E. Fleharty, F. van Swol, D. N. Petsev, The effect of surface charge regulation on conductivity in fluidic nanochannels, J. Colloid Interface Sci. 416 (2014) 105-111.

[47] M. E. Fleharty, F. van Swol, D. N. Petsev, Manipulating semiconductor colloidal stability through doping, Phys. Rev. Lett. 113 (2014) 158302.

[48] E. Greenfield, U. Sivan, Measuring changes in surface potential as two charged bodies approach in electrolyte solution, Phys. Rev. Lett. 102 (2009) 106101.

[49] G. Trefal, S. H. Behrens, M. Borkovec, Charge regulation in the electrical double layer: Ion adsorption and surface interactions, Langmuir 32 (2016) 380-400.

[50] W. Kunz, J. Henle, B. W. Ninham, The present state of affairs with hofmeister effects, Curr. Opin. Colloid Interface Sci. 9 (2004) 1-18.

[51] J. H. W. Kunz, B. Ninham, 'zur lehre von der wirkung der salze' (about the science of the effect of salts): Franz hofmeister's historical papers, Current Opinion in Colloid and Interface Science 9 (2004) 19-37.

[52] T. F. Tadros, J. Lyklema, Adsorption of potential-determining ions at the silica-aqueous electrolyte interface and the role of some cations, J. Electroanal. Chem. 17 (1968) 265-275.

[53] I. U. Vakarelski, K. Ishimura, K. Higashitani, Adhesion between silica particle and mica surfaces in water and electrolyte solutions, J. Colloid Interface Sci. 227 (2000) 111-118.

[54] I. U. Vakarelski, K. Higashitani, Dynamic features of short-range interaction force and adhesion in solutions, J. Colloid Interface Sci. 242 (2001) $110-120$.

[55] B. C. B. C. Donose, I. U. Vakarelski, K. Higashitani, Silica surfaces lubrication by hydrated cations adsorption from electrolyte solutions, Lang- 
muir 21 (2005) 1834-1839.

2 [56] S. S. Dukhin, B. V. Derjaguin, Equilibrium double layer and electrokinetic phenomena, in: E. Matijevic (Ed.), Surface and Colloid Science, Ch. 2 .

[57] R. J. Hunter, Zeta Potential in Colloid Science, Academic Press, New York, 1981.

[58] W. B. Russel, D. A. Saville, W. R. Schowalter, Colloidal Dispersions, Cambridge Univsersity Press, New York.

[59] R. Qiao, N. R. Aluru, Ion concentrations and velocity profiles in nanochannel electroosmotic flows, J. Chem. Phys. 118 (2003) 46924701.

60] R. Qiao, N. R. Aluru, Charge inversion and flow reversal in a nanochannel electro-osmotic flow, Phys. Rev. Lett. 92 (2004) 198301.

[61] R. Qiao, N. R. Aluru, Scaling of electrokinetic transport in nanometer channels, Langmuir 21 (2005) 8972-8977.

[62] P. Wu, R. Qiao, Physical origins of apparently enhanced viscosity of interfacial fluids in electrokinetic transport, Phys. Fluids 23 (2011) 072005.

[63] A. Thompson, Nonequilibrium molecular dynamics simulation of electroosmotic flow in a charged nanopore, J. Chem. Phys. 119 (2003) 75037511.

[64] Sandia National Laboratories Tramonto Software: https://software.Sandia.Gov/tramonto/.

[65] S. Garikipati, Evaluation of colloidal titration for the determination of surface charge of activated sludge flocs, Master's thesis, Chalmers University OF Technology (2005).

[66] J. W. Morgan, C. F. Forster, L. Evison, A comparative study of the nature of biopolymers extracted from anaerobic and activated sludges, Wat. Res. 24 (1990) 743-750.

[67] L. H. Mikkelsen, Applications and limitations of the colloid titration method for measuring activated sludge surface charges, Wat. Res. 37 (2003) 2458-2466.

[68] C. Labbez, B. Jo1nsson, I. Pochard, A. Nonat, B. Cabane, Surface charge density and electrokinetic potential of highly charged minerals: Experiments and monte carlo simulations on calcium silicate hydrate, J. Phys. Chem. B 110 (2006) 9219-9230.

[69] J. M. G. Barthel, H. Krienke, Physical Chemistry of Electrolyte Solutions: Modern Aspects, Springer.

[70] R. E. G. van Hal, J. C. T. Eijkel, P. Bergveld, A general model to describe the electrostatic potential at electrolyte oxide interfaces, Adv. Colloid Interface Sci. 69 (1996) 31-62.

[71] P. Debye, E. Huckel, Zur theorie der elektrolyte. i. gefrierpunktserniedrigung und verwandte erscheinungen, Phys. Ztschr. 24 (1923) 185-206.

[72] G. A. Martynov, Power and exponential asymptotic forms of correlation functions, Theoretical and Mathematical Physics 156 (2008) 1356-1364.

[73] J. N. Israelachvili, Intermolecular and Surface Forces. 3rd edn, Academic Press, 2011.

[74] H. Löwen, M. Heinen, Dynamical density functional theory for the diffusion of injected brownian particles, Eur. Phys. J. Special Topics 223 (2014) 1-15. 

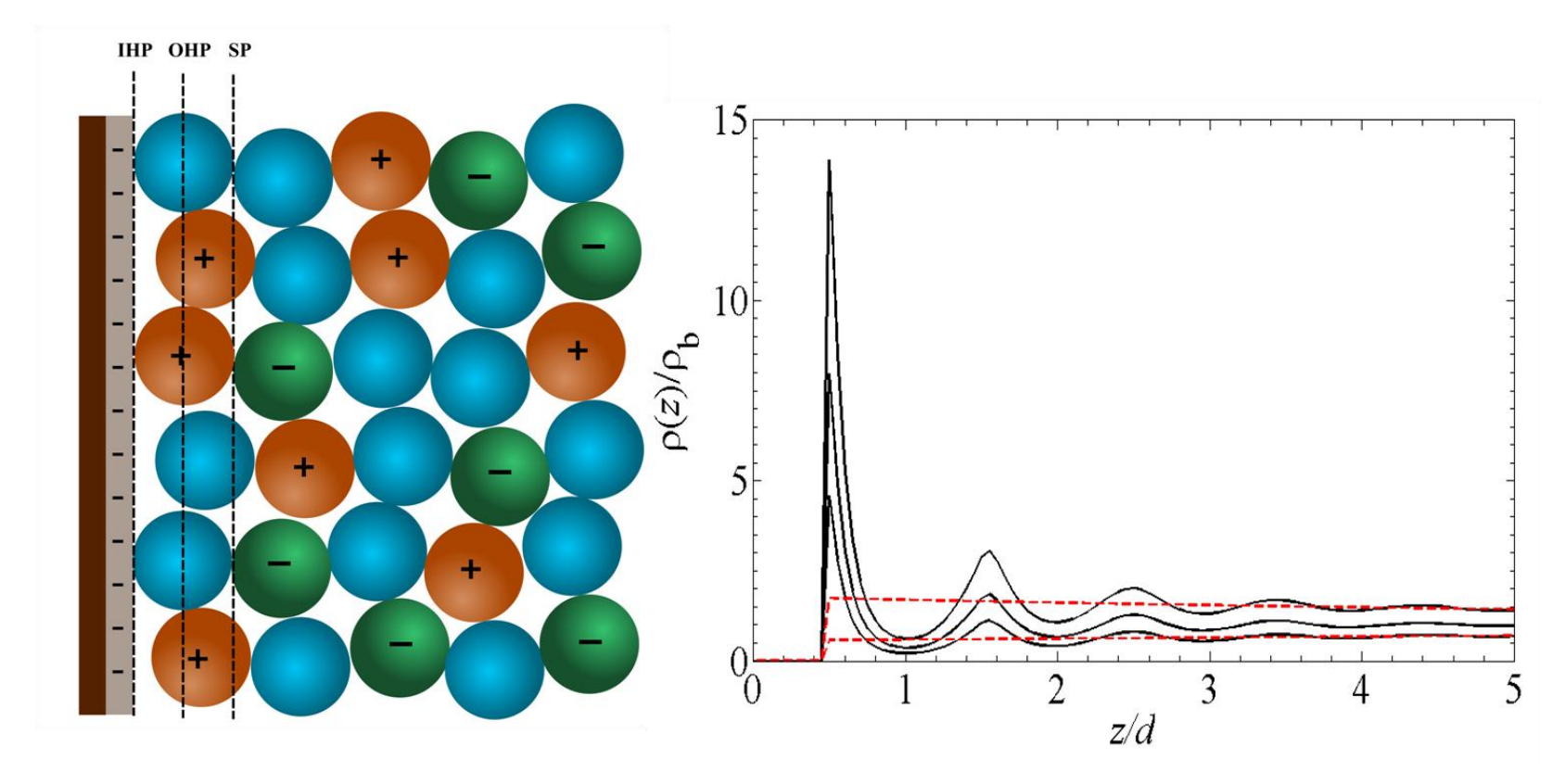

\section{Graphical Abstract}

(n)

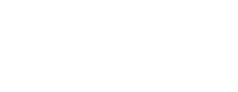

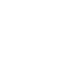

- 